Obere Extremität 2022 $17: 1-2$

https://doi.org/10.1007/s11678-022-00681-0 Angenommen: 3. Februar 2022

(C) The Author(s), under exclusive licence to Springer Medizin Verlag GmbH, ein Teil von Springer Nature 2022

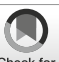

\title{
Wechsel in der Schriftleitung der Zeitschrift Obere Extremität
}

\author{
Kilian Wegmann ${ }^{1} \cdot$ Christoph Katthagen $^{2} \cdot$ Philipp Moroder ${ }^{3}$ \\ ${ }^{1}$ Klinik und Poliklinik für Orthopädie und Unfallchirurgie, Universitätsklinikum Köln, Köln, Deutschland \\ ${ }^{2}$ Klinik für Unfall-, Hand- und Wiederherstellungschirurgie, Universitätsklinikum Münster, Münster, \\ Deutschland \\ ${ }^{3}$ Schulthess Klinik Zürich, Zürich, Schweiz
}

Sehr geehrte Leserinnen und Leser,

wir hoffen Sie sind gut in das neue Jahr 2022 gestartet. Mit dem Wechsel der Schriftleitung hat das neue Jahr auch bei der ObEx eine Veränderung gebracht. Mit dem Ausklang des Jahres 2021 haben Lars Müller, Helmut Lill und Markus Scheibel ihre Rolle als Schriftleitung der ObEx abgegeben. Durch ihr stetiges und innovatives Wirken wurde die wissenschaftliche Ausrichtung der Zeitschrift gestärkt und das Erscheinungsbild regelmäßig aufgewertet und verbessert. Ohne das Wirken der Schriftleitung, unserer Vorgänger, wäre die ObEx mit Sicherheit nicht auf dem heutigen Niveau.

Gemeinsam haben wir in den letzten Jahren intensivste Bemühungen unternommen, die ObEx als wissenschaftliche Zeitschrift in Medline zu listen. Auch wenn dieses Unterfangen nicht geglückt ist, wurde hierdurch das Gesicht der ObEx geformt und die Qualität der Beiträge verbessert.
Lieber Markus, lieber Helmut, lieber Lars, wir wollen Euch in unserem Namen, im Namen der Zeitschriftenredaktion und im Namen aller Leserinnen und Leser unseren herzlichen Dank aussprechen! Wir sind sehr froh und dankbar, dass Ihr mit Eurer Expertise und Eurem Wissen der ObEx im Rahmen der Herausgeberschaft weiter verbunden bleiben werdet.

Als neue Schriftleitung (• Abb. 1) stehen wir großen Herausforderungen gegenüber. Auch wenn es ein lang gehegter Wunsch der Herausgeber und der Schriftleitung war, wird es auf Grund von neuen Vorgaben der bestimmenden Institution in Zukunft nicht mehr möglich sein, eine Listung von teils deutschsprachigen Zeitschriften ohne Fokus auf wissenschaftliche Originalarbeiten bei Medline zu erreichen. Aufgrund des Feedbacks unserer Leserinnen und Leser sind wir jedoch nicht bereit die ObEx den Medline-Vorgaben entsprechend in eine rein englischsprachige Zeitschrift mit Fokus auf Originalarbeiten zu entwickeln. Gemeinsam haben wir uns entschlossen, die ObEx schrittweise so-

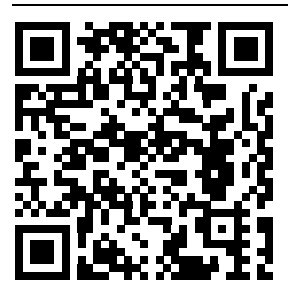

QR-Code scannen \& Beitrag online lesen
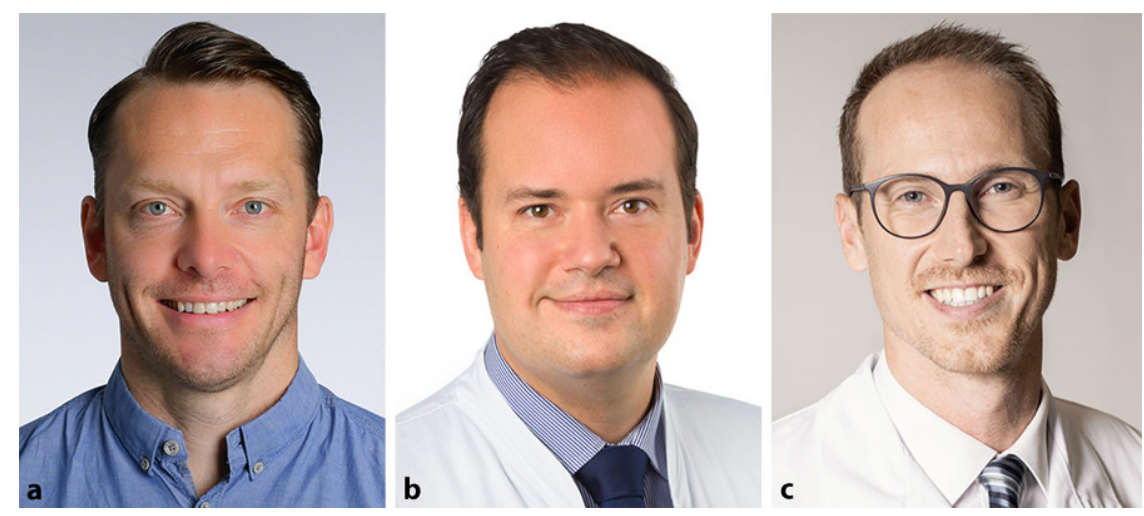

Abb. $1 \Delta$ Die neue Schriftleitung: Prof. Dr. K. Wegmann, Prof. Dr. C. Katthagen, Prof. Dr. P. Moroder 


\section{Editorial}

wohl sprachlich als auch inhaltlich wieder näher an die Bedürfnisse ihrer Leserinnen und Leser innerhalb der D-A-CH-Region heranzuführen. Mit Ausnahme von ausgewählten internationalen Gastbeiträgen werden die Artikel der ObEx auf Deutsch erscheinen und das Hauptaugenmerk wird auf Übersichtsartikeln liegen, die für den klinischen Alltag nützlich sein können. Zusätzlich werden wir die Bereiche der praxisnahen Fort- und Weiterbildung ausbauen mit gezielter Förderung der jungen Leserinnen und Leser der ObEx und der Mitglieder der DVSE. Dies soll durch Rubriken, die sich mit den täglichen Problemen des Berufsalltages - wie z.B. der Abrechnung und medikolegalen Aspekten - beschäftigen, erreicht werden. Zudem möchten wir die Präsenz der ObEx in den sozialen Medien stärken, um die Reichweite zu erhöhen. Um diese neuen Aufgaben adäquat abdecken zu können, wurden die jeweiligen Bereiche mit Rubriken-Herausgebern besetzt. Wir sind froh und stolz darauf, ein Spitzenteam um uns zu wissen, das uns bei der spannenden Arbeit für die ObEx unterstützen und mit uns das Ziel verfolgen wird, Ihnen weiterhin eine hochwertige und interessante Zeitschrift bieten zu können.

\section{Korrespondenzadresse}

Prof. Dr. med. Kilian Wegmann

Klinik und Poliklinik für Orthopädie und Unfallchirurgie, Universitätsklinikum Köln Kerpenerstr. 62, 50931 Köln, Deutschland Kilian.Wegmann@uk-koeln.de

Interessenkonflikt. K. Wegmann, C. Katthagen und P. Moroder geben an, dass kein Interessenkonflikt besteht. 